\title{
Two-electron capture with emission of one photon in fast collisions between a highly charged ion and a light atom
}

\author{
A.B.Voitkiv ${ }^{1,2}$, B.Najjari ${ }^{1}$, N.Toshima ${ }^{2}$ and J.Ullrich ${ }^{1}$ \\ ${ }^{1}$ Max-Planck-Institut für Kernphysik, \\ Saupfercheckweg 1, D-69117 Heidelberg, Germany \\ 2 Institute for Materials Science, University of Tsukuba, \\ 1-1-1 Tennodai, Tsukuba, Ibaraki 305 8573, Japan
}

April 12, 2006

\begin{abstract}
We consider the electron capture process in fast non-relativistic ion-atom collisions in which the transfer of two electrons from the atom to the ion is accompanied by emission of one photon with the mean energy $\omega_{k}^{(2)}$ about two times larger than that $\omega_{k}^{(1)}$ characteristic for the radiative capture of one electron. Such a photon can appear both due to the uncorrelated capture process, in which two electrons are transferred to the ion independently via the Coulomb and radiative capture channels, and due to the correlated two-electron capture, where the electron-electron interaction plays the crucial role. The uncorrelated capture produces the photon spectrum which has a maximum at $\omega_{k}^{(1)}$ and gives the main contribution to the two-electron capture. The correlated capture mechanism leads to very small capture cross sections but produces the photon spectrum having a maximum at $\sim \omega_{k}^{(2)}$ which in principle enables to separate this process in experiment.
\end{abstract}

PACS: $34.70 .+\mathrm{e}$ 


\section{Introduction}

Transfer of electrons between colliding ion and atom represents one of the basic processes studied in atomic physics. In fast ion-atom collisions the most important capture process having largest cross sections is the one-electron capture.

There are two distinct processes resulting in the capture of one atomic electron by an incident ion. In the first of them the electron is transferred to the ion due to the Coulomb interaction in the system of rearranging atomic particles. Such a process is usually referred to as the Coulomb (or nonradiative) capture.

In the other capture process the coupling of the colliding ion-atom system to the radiation field is of great importance. In this process, which is called the radiative capture, the collision 'triggers' the interaction of the electron with its own electromagnetic field and the electron capture proceeds with emission of a real photon. Although the coupling 'constant' for the interaction between the electron and its own field is per se quite weak, the radiative capture channel may become rather effective because of its resonance nature. Indeed, the photon emission results in the matching of the minimum momentum transfer in the collision to the velocity of the ion which, at high collision velocities, drastically improves the kinematic conditions for the electron transfer and can make the capture process more likely to proceed with than without emission of a photon.

In the 'ordinary' radiative capture process only one electron is transferred to the ion and one photon is emitted. This process has been studied extensively for many years and a large amount of both experimental and theoretical results for this process covering collision energies from several $\mathrm{MeV} / \mathrm{u}$ up to $160 \mathrm{GeV} / \mathrm{u}$ is available in the literature (see, for instance, [1]- [17] and references therein).

Besides the 'ordinary' radiative capture there also exists a more complicated capture process in which two electrons are transferred from the atom to the ion in a single collision event but only one photon is emitted during the process. First efforts to study this process were undertaken more than ten years ago [18]. Theoretically the radiative capture of two electrons with emission of a one photon was addressed in [19] and [20] where this process was regarded as the radiative recombination of two free electrons which, in the initial channel, move with respect to the ion with equal velocities.

The present article is an attempt to consider the process of the two-electron capture with emission of one photon as a two-center ion-atom collision phenomenon. Atomic units are used throughout the paper except where otherwise stated.

\section{Theoretical framework}

In the consideration of the capture in this article we shall adopt the semi-classical approach. Within this approach the electrons and the electromagnetic field are described as quantum objects while the heavy nuclei of the colliding ion and atom are regarded as classical particles. We shall assume that the non-relativistic electrons are initially bound 
in an internal state $\varphi_{i}$ of the atom-target with the nucleus charge $Z_{t}$. As a result of the collision between the atom and a nucleus-projectile with a charge $Z_{p}$ the electrons are captured into an internal state $\psi_{f}$ of the ion-projectile and a photon is emitted. Because of comparatively very large masses of the nuclei, the coupling of the nuclei to the radiation field is much weaker than that of the electrons and will be neglected.

We shall consider the REC process in the rest frame of the incident nucleus (the projectile frame) and take the position of the nucleus as the origin of this frame. In the projectile frame the atomic nucleus is assumed to move along a classical straight-line trajectory $\mathbf{R}(t)=\mathbf{b}+\mathbf{v} t$, where $\mathbf{b}$ is the impact parameter of the atom with respect to the origin and $\mathbf{v}$ the atomic velocity. The coordinates of the electrons with respect to the origin are denoted by $\mathbf{s}_{1}$ and $\mathbf{s}_{2}$. The coordinates of the electrons with respect to the nucleus of the atom are $\mathbf{r}_{1}$ and $\mathbf{r}_{2}$. Note that $\mathbf{r}_{1,2}=\mathbf{s}_{1,2}-\mathbf{R}$.

Within the semi-classical approach, the transition amplitude as a function of the impact parameter, obtained in the first order approximation in the interaction between the electrons and the electromagnetic field, is given by

$$
a_{f i}(\mathbf{b})=-i \int_{-\infty}^{+\infty} d t\left\langle\Psi_{f}(t)\left|\hat{W}_{E M}\right| \Psi_{i}(t)\right\rangle
$$

Here

$$
\hat{W}_{E M}=-\frac{e}{2 m c} \sum_{j=1,2}\left(\hat{\mathbf{p}}_{j} \cdot \hat{\mathbf{A}}_{j}+\hat{\mathbf{A}}_{j} \cdot \hat{\mathbf{p}}_{j}\right)
$$

is the interaction between the electrons and the electromagnetic field, where $\hat{\mathbf{p}}_{j}(j=1,2)$ is the momentum operator of the $j$-th electron, $e$ is the electron charge, $m$ its mass, $c=137$ a.u. is the speed of light and $\hat{\mathbf{A}}_{j}$ is the vector potential of the quantized electromagnetic field in the point $\mathbf{s}_{j}$. The potential reads

$$
\hat{\mathbf{A}}(\mathbf{s})=\sum_{\mathbf{k}^{\prime} \lambda^{\prime}} \sqrt{\frac{2 \pi c^{2}}{V \omega_{k}^{\prime}}} \mathbf{e}_{\mathbf{k}^{\prime} \lambda^{\prime}}\left(c_{\mathbf{k}^{\prime} \lambda^{\prime}}^{+} \exp \left(i \omega_{k}^{\prime} t-i \mathbf{k}^{\prime} \cdot \mathbf{s}\right)+c_{\mathbf{k}^{\prime} \lambda}^{-} \exp \left(i \mathbf{k}^{\prime} \cdot \mathbf{s}-i \omega_{k}^{\prime} t\right)\right),
$$

where $c_{\mathbf{k}^{\prime} \lambda^{\prime}}^{+}$and $c_{\mathbf{k}^{\prime} \lambda^{\prime}}^{-}$are the creation and destruction operators, respectively, for a photon with a momentum $\mathbf{k}^{\prime}$, polarization $\mathbf{e}_{\mathbf{k}^{\prime} \lambda^{\prime}}\left(\lambda^{\prime}=1,2\right)$ and frequency $\omega_{k}^{\prime}=c\left|\mathbf{k}^{\prime}\right|$, and $V$ is the normalization volume for the field. The sum in Eq.(3) runs over all photon modes.

The initial and final states, $\left|\Psi_{i}(t)\right\rangle$ and $\left|\Psi_{f}(t)\right\rangle$, read

$$
\begin{aligned}
\left|\Psi_{i}(t)\right\rangle & =\Phi_{i}(t)|0\rangle \\
\left|\Psi_{f}(t)\right\rangle & =\Phi_{f}(t)|\mathbf{k} \lambda\rangle .
\end{aligned}
$$

Here, $\Phi_{i}^{(+)}\left(\mathbf{s}_{1}, \mathbf{s}_{2}, t\right)$ and $\Phi_{f}^{(-)}\left(\mathbf{s}_{1}, \mathbf{s}_{2}, t\right)$ are solutions of the time-dependent Schrödinger equation

$$
i \frac{\partial}{\partial t} \Phi(t)=\left(\hat{H}_{1}+\hat{H}_{2}+\frac{1}{s_{12}}\right) \Phi(t)
$$


with appropriate boundary conditions, where

$$
\hat{H}_{j}(t)=\frac{\hat{\mathbf{p}}_{j}^{2}}{2}-\frac{Z_{t}}{r_{j}}-\frac{Z_{p}}{s_{j}} ; j=1,2
$$

are the one-electron Hamiltonians. Further $|0\rangle$ is the vaccuum state of the radiation field and $|\mathbf{k} \lambda\rangle$ is the state of this field containing one photon with a momentum $\mathbf{k}$, polarization $\mathbf{e}_{\mathbf{k} \lambda}$ and frequency $\omega_{k}$.

If the change in the electron momentum in the capture process is much less than $m c=137$ a.u., the contribution to the capture from electron spin-flip transitions is of minor importance and can be neglected. Within such an approximation projections of electron spins do not change in the collision and, as a result, the (a)symmetry of the space part of the two-electron states is conserved in the capture process. Below we shall restrict our consideration only to the capture to the $K$-shell. This, in particular, means that the space part of the electron wavefunction is symmetric in both the initial and final states.

In order to get some basic ideas about the possible mechanisms of the two-electron capture with emission of one photon, let us assume for the moment that we know exact solutions $\Phi_{i}^{(+)}\left(\mathbf{s}_{1}, \mathbf{s}_{2}, t\right)$ and $\Phi_{f}^{(-)}\left(\mathbf{s}_{1}, \mathbf{s}_{2}, t\right)$ of (5) which describe the motion of the electrons in the combined field of the colliding nuclei of the atom and ion. These states satisfy the corresponding 'in' and 'out' boundary conditions, $\Phi_{i}^{(+)}(t \rightarrow-\infty) \rightarrow \varphi_{i} \exp \left(-i \epsilon_{i} t\right)$ and $\Phi_{f}^{(-)}(t \rightarrow+\infty) \rightarrow \psi_{f} \exp \left(-i \varepsilon_{f} t\right)$, respectively. Since these states are exact, their scalar product $\left\langle\Phi_{f}^{(-)}(t) \mid \Phi^{(+)}(t)\right\rangle$ is time-independent

$$
\frac{\partial}{\partial t}\left(\left\langle\Phi_{f}^{(-)}(t) \mid \Phi^{(+)}(t)\right\rangle\right)=0
$$

and represents the amplitude of the non-radiative (Coulomb) capture.

With these states the transition amplitude for the radiative electron capture, which is of the first order in the interaction with the radiation field but otherwise exact, reads

$$
\begin{aligned}
a_{f i}(\mathbf{b})= & \frac{i e}{m c} \int_{-\infty}^{+\infty} d t \exp \left(i \omega_{k} t\right) \times \\
& \left\langle\Phi_{f}^{(-)}(t)\left|\exp \left(-i \mathbf{k} \cdot \mathbf{r}_{1}\right) \boldsymbol{\alpha}_{\mathbf{k} \lambda} \cdot \hat{\mathbf{p}}_{1}+\exp \left(-i \mathbf{k} \cdot \mathbf{r}_{2}\right) \boldsymbol{\alpha}_{\mathbf{k} \lambda} \cdot \hat{\mathbf{p}}_{2}\right| \Phi_{i}^{(+)}(t)\right\rangle
\end{aligned}
$$

where $\boldsymbol{\alpha}_{\mathbf{k} \lambda}=\sqrt{\frac{2 \pi c^{2}}{V \omega_{k}}} \mathbf{e}_{\mathbf{k} \lambda}$.

If the electron-electron interaction is neglected (and for simplicity the electrons are assumed to be distinguishable) the two-electron states can be presented as

$$
\begin{aligned}
& \Phi_{i}^{(+)}(t)=\phi_{i, 1}^{(+)}\left(\mathbf{r}_{1}, t\right) \chi_{i, 2}^{(+)}\left(\mathbf{r}_{2}, t\right) \\
& \Phi_{f}^{(-)}(t)=\phi_{f, 1}^{(-)}\left(\mathbf{r}_{1}, t\right) \chi_{f, 2}^{(-)}\left(\mathbf{r}_{2}, t\right),
\end{aligned}
$$

where $\phi^{( \pm)}(t)$ and $\chi^{( \pm)}(t)$ are exact solutions of the corresponding one-electron Schrödinger equations with the Hamiltonians $\hat{H}_{1}$ and $\hat{H}_{2}$, respectively, obeying appropriate 'in' and 
'out' boundary conditions. For these states one has

$$
\begin{aligned}
& \frac{\partial}{\partial t}\left(\left\langle\phi_{f, 1}^{(-)}(t) \mid \phi_{i, 1}^{(+)}(t)\right\rangle\right)=0 \\
& \frac{\partial}{\partial t}\left(\left\langle\chi_{f, 2}^{(-)}(t) \mid \chi_{i, 2}^{(+)}(t)\right\rangle\right)=0
\end{aligned}
$$

which is exactly similar to that of Eq.(7), and their time-independent scalar products, $\left\langle\phi_{f, 1}^{(-)} \mid \phi_{i, 1}^{(+)}\right\rangle$and $\left\langle\chi_{f, 2}^{(-)} \mid \chi_{i, 2}^{(+)}\right\rangle$, are the amplitudes for the non-radiative capture of the first and second electron.

Inserting the states (9) into the transition amplitude (8) and taking into account (10) we find that

$$
\begin{aligned}
a_{f i}(\mathbf{b})= & \frac{i e}{m c} \times \\
& \left(\left\langle\phi_{f, 1}^{(-)} \mid \phi_{i, 1}^{(+)}\right\rangle \int_{-\infty}^{+\infty} d t \exp \left(i \omega_{k} t\right)\left\langle\chi_{f, 2}^{(-)}(t)\left|\exp \left(-i \mathbf{k} \cdot \mathbf{r}_{2}\right) \boldsymbol{\alpha}_{\mathbf{k} \lambda} \cdot \hat{\mathbf{p}}_{2}\right| \chi_{i, 2}^{(+)}(t)\right\rangle\right. \\
& \left.+\left\langle\chi_{f, 2}^{(-)} \mid \chi_{i, 2}^{(+)}\right\rangle \int_{-\infty}^{+\infty} d t \exp \left(i \omega_{k} t\right)\left\langle\phi_{f, 1}^{(-)}(t)\left|\exp \left(-i \mathbf{k} \cdot \mathbf{r}_{1}\right) \boldsymbol{\alpha}_{\mathbf{k} \lambda} \cdot \hat{\mathbf{p}}_{1}\right| \phi_{i, i}^{(+)}(t)\right\rangle() 1\right)
\end{aligned}
$$

The physical meaning of the amplitude (11) is simple: it is the product of the amplitude for the non-radiative capture of one electron and the amplitude for the radiative capture of the other electron. The amplitude (11) is not equal to zero which shows that the capture of two electrons with the emission of one photon is possible without the electronelectron interaction (or the electron-electron correlation). However, since in the physical mechanism of the capture, described by the amplitude (11), only one electron is transferred due to the coupling to the radiation field, the photon spectrum calculated with (11) has a maximum at a frequency $\omega_{k}^{(1)} \sim\left(v^{2} / 2+Z_{p}^{2} / 2\right)$ which is typical for the 'ordinary' (one-electron) radiative capture. As a result, if we calculate the emission of a photon with a double frequency $\omega_{k}^{(2)} \sim\left(v^{2}+Z_{p}^{2}\right)$ using the uncorrelated amplitude (11), we find that this frequency lies very far from the maximum of the calculated photon spectrum and that this spectrum at $\omega_{k} \sim \omega_{k}^{(2)}$ just monotonously decreases with the frequency increase. Therefore, any particular structure found in the photon spectrum in the region of $\omega_{k} \sim\left(v^{2}+Z_{p}^{2}\right)$ (for instance, a maximum) has to be attributed solely due to effect of the electron-electron interaction.

Below we shall consider the correlated and uncorrelated capture processes separately.

\subsection{Correlated two-electron capture}

In what follows it will be more convenient to work with the transition amplitude in the momentum space, $S_{f i}\left(\mathbf{Q}_{\perp}\right)$, which is related to the amplitude (1) by the two-dimensional Fourier transformation

$$
S_{f i}\left(\mathbf{Q}_{\perp}\right)=\frac{1}{2 \pi} \int d^{2} \mathbf{b} a_{f i}(\mathbf{b}) \exp \left(i \mathbf{Q}_{\perp} \cdot \mathbf{b}\right) .
$$


This transition amplitude will be evaluated in this subsection using three different models for the radiative capture.

\subsubsection{First Born approximation}

Within this approximation the initial and final states are taken as

$$
\begin{aligned}
& \left|\Psi_{i}^{(+)}(t)\right\rangle=\exp \left(-i\left(\epsilon_{i}+v^{2}\right) t\right) \exp \left(i \mathbf{v} \cdot\left(\mathbf{s}_{1}+\mathbf{s}_{2}\right)\right) \varphi_{i}\left(\mathbf{r}_{1}, \mathbf{r}_{2}\right)|0\rangle \\
& \left|\Psi_{f}^{(-)}(t)\right\rangle=\exp \left(-i \varepsilon_{f} t\right) \psi_{f}\left(\mathbf{s}_{1}, \mathbf{s}_{2}\right)|\mathbf{k} \lambda\rangle
\end{aligned}
$$

Here $\varphi_{i}\left(\mathbf{r}_{1}, \mathbf{r}_{2}\right)$ is the initial state of the electrons, which is approximated by an undistorted eigenstate of the atom, and $\psi_{f}\left(\mathbf{s}_{1}, \mathbf{s}_{2}\right)$ is the final state of the electrons which is taken as an undistorted eigenstate of the ion. The initial and final energies of the electrons are given by $\epsilon_{i}+v^{2}$ and $\varepsilon_{f}$, respectively, where $\epsilon_{i}$ is the electron binding energy in the atom and $\varepsilon_{f}$ is that in the ion. Further, $|0\rangle$ denotes the electromagnetic field vacuum and $|\mathbf{k} \lambda\rangle$ is the photon state with one photon which has momentum $\mathbf{k}$, polarization $\lambda$ and frequency $\omega_{k}=c|\mathbf{k}|=c k$.

Taking into account Eqs.(1)-(13) and the symmetry of the states $\varphi_{i}\left(\mathbf{r}_{1}, \mathbf{r}_{2}\right)$ and $\psi_{f}\left(\mathbf{s}_{1}, \mathbf{s}_{2}\right)$ $\left(\varphi_{i}\left(\mathbf{r}_{1}, \mathbf{r}_{2}\right)=\varphi_{i}\left(\mathbf{r}_{2}, \mathbf{r}_{1}\right), \psi_{f}\left(\mathbf{s}_{1}, \mathbf{s}_{2}\right)=\psi_{f}\left(\mathbf{s}_{2}, \mathbf{s}_{1}\right)\right)$ the transition amplitude in the momentum space can be presented in the following form

$$
S_{f i}^{1 B}\left(\mathbf{Q}_{\perp}\right)=\frac{8 \pi^{2} i}{c v} \int d^{3} \mathbf{p} \phi_{i}(\mathbf{p}, \mathbf{q}-\mathbf{p}) \chi_{f}^{*}(\mathbf{p}+\mathbf{v}-\mathbf{k}, \mathbf{v}+\mathbf{q}-\mathbf{p})\left(\boldsymbol{\alpha}_{\mathbf{k} \lambda} \cdot(\mathbf{p}+\mathbf{v})\right)
$$

In the above expression $\phi_{i}\left(\mathbf{p}_{1}, \mathbf{p}_{2}\right)\left(=\phi_{i}\left(\mathbf{p}_{2}, \mathbf{p}_{1}\right)\right)$ and $\chi_{f}\left(\mathbf{p}_{1}, \mathbf{p}_{2}\right)\left(=\chi_{f}\left(\mathbf{p}_{2}, \mathbf{p}_{1}\right)\right)$ are the Fourier transforms of the initial and final states $\varphi_{i}\left(\mathbf{r}_{1}, \mathbf{r}_{2}\right)$ and $\psi_{f}\left(\mathbf{s}_{1}, \mathbf{s}_{2}\right)$, respectively. Further, $\mathbf{q}=\left(\mathbf{Q}_{\perp}, q_{m}\right)$ is the momentum transfer in the collision where $\mathbf{Q}_{\perp}$ is its transverse part, $\mathbf{Q}_{\perp} \cdot \mathbf{v}=0$, and

$$
q_{m}=\frac{\omega_{k}+\varepsilon_{f}-\epsilon_{i}}{v}-v
$$

is the minimum momentum transfer.

\subsubsection{Continuum-Distorted-Wave approximation}

In order to improve the description of the electrons in the initial and final states, in both of which the electrons move in the combined long-range Coulomb fields of the nuclei, we employ the Continuum Distorted Wave (CDW) approximation which has been successfully used in the theory of fast non-relativistic ion-atom collisions (see e.g. [21], [22]).

Within the CDW approximation the initial and final states are taken as

$$
\begin{aligned}
\left|\Psi_{i}^{(+)}(t)\right\rangle & =\exp \left(-i\left(\epsilon_{i}+v^{2}\right) t\right) L_{i}^{(+)}\left(\mathbf{s}_{1}\right) L_{i}^{(+)}\left(\mathbf{s}_{2}\right) \exp \left(i \mathbf{v} \cdot\left(\mathbf{s}_{1}+\mathbf{s}_{2}\right)\right) \varphi_{i}\left(\mathbf{r}_{1}, \mathbf{r}_{2}\right)|0\rangle \\
\left|\Psi_{f}^{(-)}(t)\right\rangle & =\exp \left(-i \varepsilon_{f} t\right) L_{f}^{(-)}\left(\mathbf{r}_{1}\right) L_{f}^{(-)}\left(\mathbf{r}_{2}\right) \psi_{f}\left(\mathbf{s}_{1}, \mathbf{s}_{2}\right)|\mathbf{k} \lambda\rangle
\end{aligned}
$$


The states (16) differ from those given in (13) by the presence of the distortion factors $L_{i}^{(+)}\left(\mathbf{s}_{1}\right), L_{i}^{(+)}\left(\mathbf{s}_{2}\right), L_{f}^{(-)}\left(\mathbf{r}_{1}\right)$ and $L_{f}^{(-)}\left(\mathbf{r}_{2}\right)$. The factors $L_{i}^{(+)}\left(\mathbf{s}_{1}\right)$ and $L_{i}^{(+)}\left(\mathbf{s}_{2}\right)$ are introduced in order to take into account the distortion of the initial bound atomic state by the ionic nucleus. Similarly, $L_{f}^{(-)}\left(\mathbf{r}_{1}\right)$ and $L_{f}^{(-)}\left(\mathbf{r}_{2}\right)$ account for the distortion of the final state of the electrons captured to the ion which is caused by the field of the atomic nucleus.

The initial and final distortion factors read

$$
\begin{aligned}
& L_{i}^{(+)}(\mathbf{s})=\exp \left(\pi \nu_{p}\right) \Gamma\left(1-i \nu_{p}\right) F\left(i \nu_{p}, 1, i v s-i \mathbf{v} \cdot \mathbf{s}\right) \\
& L_{f}^{(-)}(\mathbf{r})=\exp \left(\pi \nu_{t}\right) \Gamma\left(1+i \nu_{t}\right) F\left(-i \nu_{t}, 1,-i v r+i \mathbf{v} \cdot \mathbf{r}\right)
\end{aligned}
$$

In Eqs.(17) $\nu_{p}=Z_{p} / v, \nu_{t}=Z_{t} / v, \Gamma$ is the gamma-function and $F(a, b, z)$ is the degenerate hypergeometric function (see e.g. [23]).

In expression (16) the distortions for the initial and final electron bound states are taken symmetrically. However, having in mind that we shall be interested only in collisions with $Z_{p} \gg Z_{t}$, the distortion factors for the final state may be replaced by 1 . Then, taking into account Eqs.(1)-(12) and (16)-(17) and the symmetry of the electronic wavefunctions, the transition amplitude can be written as

$$
\begin{aligned}
S_{f i}^{C D W}\left(\mathbf{Q}_{\perp}\right)= & \frac{8 \pi^{2} i}{c v} \int d^{3} \mathbf{p} \phi_{i}(\mathbf{p}, \mathbf{q}-\mathbf{p}) \\
& \times \int d^{3} \mathbf{s}_{1} \int d^{3} \mathbf{s}_{2} \psi_{f}^{*}\left(\mathbf{s}_{1}, \mathbf{s}_{2}\right) \exp \left(i(\mathbf{q}-\mathbf{p}) \cdot \mathbf{s}_{2}\right) \psi_{\mathbf{v}}^{(+)}\left(\mathbf{s}_{2}\right) \\
& \times \exp \left(-i \mathbf{k} \cdot \mathbf{s}_{1}\right)\left(\boldsymbol{\alpha}_{\mathbf{k} \lambda} \cdot \hat{\mathbf{p}}_{1}\left(\exp \left(i \mathbf{p} \cdot \mathbf{s}_{1}\right) \psi_{\mathbf{v}}^{(+)}\left(\mathbf{s}_{1}\right)\right)\right)
\end{aligned}
$$

where

$$
\psi_{\mathbf{v}}^{(+)}(\mathbf{s})=\frac{1}{(2 \pi)^{3 / 2}} \exp (i \mathbf{v} \cdot \mathbf{s}) L_{i}^{(+)}(\mathbf{s})
$$

is the incoming Coulomb wave modeling the response of the incident electron, bound in the atom, to the presence of the field of the ion nucleus.

\subsubsection{Impulse approximation}

The CDW approach of the previous subsection can be modified in the spirit of the so called impulse approximation (IA). To this end we take the initial and final states as

$$
\begin{aligned}
\left|\Psi_{i}^{(+)}(t)\right\rangle & =\exp \left(-i\left(\epsilon_{i}+v^{2}\right) t\right) \hat{L}_{i}^{(+)}\left(\mathbf{s}_{1}\right) \hat{L}_{i}^{(+)}\left(\mathbf{s}_{2}\right)\left(\exp \left(i \mathbf{v} \cdot\left(\mathbf{s}_{1}+\mathbf{s}_{2}\right)\right) \varphi_{i}\left(\mathbf{r}_{1}, \mathbf{r}_{2}\right)\right)|0\rangle \\
\left|\Psi_{f}^{(-)}(t)\right\rangle & =\exp \left(-i \varepsilon_{f} t\right) \psi_{f}\left(\mathbf{s}_{1}, \mathbf{s}_{2}\right)|\mathbf{k} \lambda\rangle .
\end{aligned}
$$

In (20) the distortion operators $\hat{L}_{i}^{(+)}\left(\mathbf{s}_{1}\right)$ and $\hat{L}_{i}^{(+)}\left(\mathbf{s}_{2}\right)$ are understood to act according to

$$
\begin{aligned}
\hat{L}_{i}^{(+)}\left(\mathbf{s}_{1}\right) \hat{L}_{i}^{(+)}\left(\mathbf{s}_{2}\right)\left(\exp \left(i \mathbf{v} \cdot\left(\mathbf{s}_{1}+\mathbf{s}_{2}\right)\right) \varphi_{i}\left(\mathbf{r}_{1}, \mathbf{r}_{2}\right)\right)= & \int d^{3} \mathbf{p}_{1} \int d^{3} \mathbf{p}_{2} \phi_{i}\left(\mathbf{p}_{1}, \mathbf{p}_{2}\right) \exp \left(-i \mathbf{p}_{1} \cdot \mathbf{R}\right) \\
& \times \exp \left(-i \mathbf{p}_{2} \cdot \mathbf{R}\right) \psi_{\mathbf{v}+\mathbf{p}_{1}}^{(+)}\left(\mathbf{s}_{1}\right) \psi_{\mathbf{v}+\mathbf{p}_{2}}^{(+)}\left(\mathbf{s}_{2}\right)(21)
\end{aligned}
$$


where $\psi_{\mathbf{v}+\mathbf{p}}^{(+)}(\mathbf{s})$ is given by (19) where the replacement $\mathbf{v} \rightarrow \mathbf{v}+\mathbf{p}$ should be made.

The main difference between the distorted states (16) and (20) lies in the replacement of the 'averaged' momentum $m \mathbf{v}=\mathbf{v}$ of the electrons with respect to the nucleus of the ion in the initial channel (this momentum enters (16)) by the 'instant' momenta of the electrons $\mathbf{v}+\mathbf{p}_{1}$ and $\mathbf{v}+\mathbf{p}_{2}$. This replacement is an attempt to take into account, in the spirit of the impulse approximation, the internal motion of the electrons in the initial state of the atom.

Using the states Eqs.(20) and the symmetry of the electronic wavefunctions the transition amplitude in the impulse approximation can be cast into the following form

$$
\begin{aligned}
S_{f i}^{I A}\left(\mathbf{Q}_{\perp}\right)=\frac{8 \pi^{2} i}{c v} & \int d^{3} \mathbf{p} \phi_{i}(\mathbf{p}, \mathbf{q}-\mathbf{p}) \int d^{3} \mathbf{s}_{1} \int d^{3} \mathbf{s}_{2} \psi_{f}^{*}\left(\mathbf{s}_{1}, \mathbf{s}_{2}\right) \\
& \times \psi_{\mathbf{v}+\mathbf{q}-\mathbf{p}}^{(+)}\left(\mathbf{s}_{2}\right) \exp \left(-i \mathbf{k} \cdot \mathbf{s}_{1}\right)\left(\boldsymbol{\alpha}_{\mathbf{k} \lambda} \cdot \hat{\mathbf{p}}_{1} \psi_{\mathbf{v}+\mathbf{p}}^{(+)}\left(\mathbf{s}_{1}\right)\right) .
\end{aligned}
$$

\subsection{Uncorrelated two-electron capture}

In the impact parameter representation the transition amplitude for the uncorrelated two-electron capture, $a_{f i}^{\text {uncor }}(\mathbf{b})$, can be written as

$$
a_{f i}^{u n c o r}(\mathbf{b})=a_{f i}^{R}(\mathbf{b}) a_{f i}^{C}(\mathbf{b}),
$$

where $a_{f i}^{R}$ is the amplitude for the radiative capture of one electron and $a_{f i}^{C}(\mathbf{b})$ is the amplitude for the Coulomb capture of the other electron.

By making use of the Fourier transformations

$$
\begin{aligned}
& a_{f i}^{R}(\mathbf{b})=\frac{1}{2 \pi} \int d^{2} \mathbf{Q}_{1} S_{f i}^{R}\left(\mathbf{Q}_{1}\right) \exp \left(-i \mathbf{Q}_{1} \cdot \mathbf{b}\right) \\
& a_{f i}^{C}(\mathbf{b})=\frac{1}{2 \pi} \int d^{2} \mathbf{Q}_{2} S_{f i}^{C}\left(\mathbf{Q}_{2}\right) \exp \left(-i \mathbf{Q}_{2} \cdot \mathbf{b}\right),
\end{aligned}
$$

where $\mathbf{Q}_{1}$ and $\mathbf{Q}_{2}$ are the two-dimensional vectors perpendicular to the collision velocity $\mathbf{v}$, the uncorrelated amplitude in the momentum space, $S_{f i}^{u n c o r}(\mathbf{Q})$, is obtained as the convolution of the corresponding radiative and Coulomb one-electron amplitudes

$$
\begin{aligned}
S_{f i}^{u n c o r}(\mathbf{Q}) & =\frac{1}{2 \pi} \int d^{2} \mathbf{b} a_{f i}^{\text {uncor }}(\mathbf{b}) \exp \left(-i \mathbf{Q}_{1} \cdot \mathbf{b}\right) \\
& =\frac{1}{2 \pi} \int d^{2} \mathbf{Q}_{1} S_{f i}^{R}\left(\mathbf{Q}_{1}\right) S_{f i}^{C}\left(\mathbf{Q}-\mathbf{Q}_{1}\right)
\end{aligned}
$$

The two-dimensional vector $\mathbf{Q}$ has the meaning of the transverse part of the total momentum transfer to the ion in the collision.

In the calculation of the uncorrelated amplitude $S_{f i}^{\text {uncor }}(\mathbf{Q})$ we evaluate the radiative and Coulomb capture amplitudes, $S_{f i}^{R}(\mathbf{Q})$ and $S_{f i}^{C}(\mathbf{Q})$, using the impulse and the CDW approximations, respectively. 


\section{Results and discussion}

\subsection{Correlated two-electron capture}

An attempt to detect in experiment the correlated two-electron capture was made in [18] using $11.4 \mathrm{MeV} / \mathrm{u}(v=21$ a.u. $) \mathrm{Ar}^{18+}$ ions colliding with a carbon target. However, no particular structure in the photon spectrum at $\omega_{k} \sim\left(v^{2}+Z_{p}^{2}\right)$ was found and, thus, the correlated process had not been observed. The authors of [18] has only estimated that the contribution of this frequency region to the two-electron capture was below $5 \mathrm{mb}$. This means that the total cross section for the correlated capture does not exceed this value.

Using the three models for the correlated capture, described in the previous section, we performed calculations for the two-electron capture into the K-shell of $11.4 \mathrm{MeV} / \mathrm{u}$ $(v=21$ a.u. $) \mathrm{Ar}^{18+}$ projectile-ions colliding with atomic helium and helium-like ions of $\mathrm{Li}, \mathrm{Be}, \mathrm{B}$ and $\mathrm{C}$. All the targets were assumed to be initially in their ground states.

Important to note that, with respect to the two-electron capture in collisions with such a highly charged ions like $\mathrm{Ar}^{18+}$, the above helium-like target ions are essentially equivalent to the corresponding neutral atoms of $\mathrm{Li}, \mathrm{Be}, \mathrm{B}$ and $\mathrm{C}$. This is because the main contribution to the two-electron capture in collisions between a highly charged ion and light targets like $\mathrm{Li}-\mathrm{C}$ is given by target K-shell electrons. Indeed, taking into account that the capture is only possible when the initial and final bound states of the electrons overlap in the space, it is not difficult to see that (in a sharp contrast to the one-electron capture) the overall probability to capture two electrons from a light target to a tightly bound state in the projectile depends inversely on the effective volume of an atomic shell where these electrons were initially localized. Therefore, in order that two electrons can be captured into such a quite compact state as the ground state of $\mathrm{Ar}^{16+}$, they have to be localized very compactly in the space also in their initial bound state. For a given atom the typical effective volume of the $L$-shell is much larger compared to that in the $K$-shell. For instance, employing Slater wavefunctions [24] for the case of carbon we find that the effective volume of its $L$ shell is roughly by a factor of 40 larger than that of the $K$-shell. Therefore, although the presence of the singlet $(2 s)^{2}$ and triplet $(2 p)^{2}$ pairs in carbon brings (in addition to the $(1 s)^{2}$ pair) seven new options to form a pair of two electrons with appropriate spin projections, their relative contribution does not exceed 20 per cent (see also the discussion of this subject in [20]).

In our calculations the initial and final electron bound states were approximated by the following wavefunctions

$$
\begin{aligned}
\varphi_{i}\left(\mathbf{r}_{1}, \mathbf{r}_{2}\right) & =N_{i} \times\left(\exp \left(-a_{i} r_{1}-b_{i} r_{2}\right)+\exp \left(-a_{i} r_{2}-b_{i} r_{1}\right)\right) \times\left(1+c_{i} \exp \left(-d_{i} r_{12}\right)\right) \\
\psi_{f}\left(\mathbf{s}_{1}, \mathbf{s}_{2}\right) & =N_{f} \times\left(\exp \left(-a_{f} s_{1}-b_{f} s_{2}\right)+\exp \left(-a_{f} s_{2}-b_{f} s_{1}\right)\right) \times\left(1+c_{f} \exp \left(-d_{f} s_{12} \gamma 26\right)\right.
\end{aligned}
$$

The variational parameters $a_{i, f}, b_{i, f}, c_{i, f}$ and $d_{i, f}$ were obtained by minimizing the energy of the corresponding ground states.

In addition to the correlated two-electron capture we performed calculations for the 


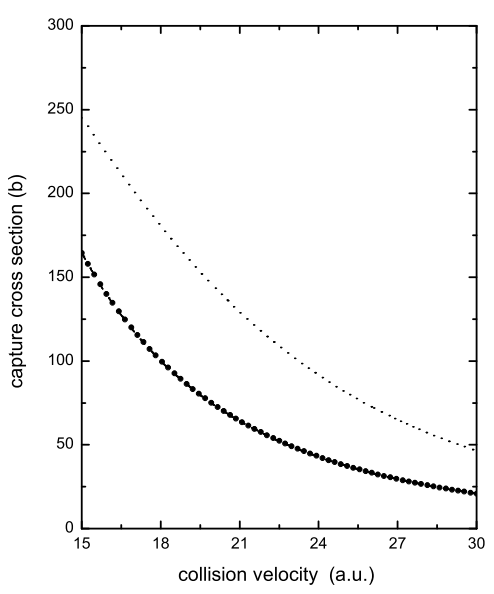

Figure 1: The cross section for the radiative capture in $\mathrm{Ar}^{18+}+\mathrm{He}\left(1 \mathrm{~s}^{2}\right) \rightarrow \mathrm{Ar}^{17+}(1 \mathrm{~s})+\mathrm{He}^{+}(1 \mathrm{~s})+$ $h \nu$ collisions given as a function of the collision velocity. Dash and dot curves display results of the CDW and first Born approximations, respectively. Solid circles show results of the impulse approximation.

one-electron radiative capture in collisions between $11.4 \mathrm{MeV} / \mathrm{u} \mathrm{Ar}^{18+}$ and He-C targets. These calculations were done using the first Born, the CDW and the impulse approximations. The results for collisions with helium are displayed in figure 1. Calculations for the other targets yielded quite close values of the cross section (per electron) and are not shown in figure 1. It is seen in the figure that the CDW and the IA lead to almost identical results for the capture cross section. Besides, in this case the first Born approximation yields cross section values which differ by no more than a factor of 2 from those given by the CDW and the IA.

The calculated results for the two-electron capture are shown in figure 2 . We see that these results turn out to be extremely strongly model-dependent. Thus, the relatively small differences between results of the different models in the case of the one-electron capture are extremely strongly enhanced in the description of the correlated two-electron capture which makes the latter a very strict test for theory.

Despite the predictions of all the models differ between themselves by orders of magnitude there nevertheless exists one feature which is common for all of them. Namely, all the models suggest that the capture cross section for collisions between a highly charged ion and very light targets increases rather rapidly with the increase in the atomic number of the target. This is a peculiarity of the two-electron capture process which is not present in the 'ordinary' radiative capture. It can be understood if one takes into account that when increasing the atomic number the target $K$-shell becomes more compressed which makes it easier to capture its two electrons into the tightly bound $K$-shell of a highly charged projectile. For the process of the one-electron capture such a feature is absent because one electron, depending on the impact parameter, can be captured by the pro- 


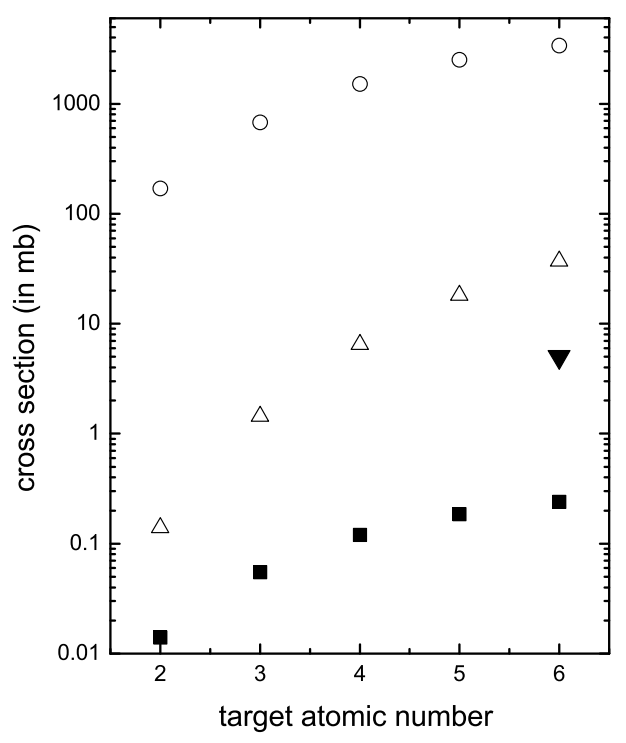

Figure 2: The section for the correlated capture of two electrons in collisions between $11.4 \mathrm{MeV} / \mathrm{u}$ $\mathrm{Ar}^{18+}$ and different targets given as a function of the atomic number of the target. Solid squares show results of the impulse approximation. Open triangles and circles display results of the CDW and first Born approximations, respectively. The solid triangle shows the experimental upper limit for the cross section.

jectile from any target shell and for the asymmetric $\left(Z_{p} \gg Z_{t}\right)$ collisions all target shells yield almost the same contribution (per electron) to the total cross section.

The difference between our first Born and CDW models lies solely in the presence of the distortion factors $L_{i}^{(+)}\left(\mathbf{s}_{1}\right)$ and $L_{i}^{(+)}\left(\mathbf{s}_{2}\right)$ in the latter. While according to the calculation of the one-electron radiative capture the role of the single distortion factor is relatively modest, the 'multiplicative' effect of two such factors turns out to be very strong, especially for the capture from the lightest targets.

On the general grounds one can expect that the CDW and IA models are superior to the simple first Born approximation. Therefore, one can conclude that in the case of the two-electron capture the Born approximation strongly fails and rule out its results.

The main physical difference between the CDW and IA models lies in the following. According to the CDW model the electron-electron correlation does not have a substantial impact on the two-electron capture. The CDW total capture cross section is essentially independent of whether the electron-electron correlation is kept in the wavefunctions (26) or it is removed by setting there $a=b$ and $c=0$. What is even more important is that the shape of the photon spectrum in the range $\omega_{k} \sim v^{2}+Z_{p}^{2}$, obtained in the CDW model, is also not sensitive to the electron correlation: this model predicts a maximum at $\omega_{k} \sim v^{2}+Z_{p}^{2}$ no matter whether the electron-electron correlation is taken into account 
or neglected ${ }^{1}$.

In a contrast, according to the IA model, the electron-electron correlation is not very important only in the initial state while the correlation in the final state becomes really crucial since without it the IA transition amplitude simply vanishes. When the electronelectron correlation is taken into account, the IA model predicts for the photon spectrum a maximum in the range $\omega_{k} \sim v^{2}+Z_{p}^{2}$.

In section II we have inferred that the electron-electron correlation is the only mechanism capable to produce a maximum in the photon spectrum at $\omega_{k} \sim v^{2}+Z_{p}^{2}$. While the IA model is in accord with this general statement, the latter is completely violated in the CDW model. Therefore, unlike in the one-electron radiative capture, these two models become quite different in the case of the correlated two-electron capture. For the latter process the IA model is clearly more physically appealing and sound and, therefore, its results are expected to be superior to those of the CDW.

This purely theoretical conclusion is supported by the data for for the two-electron capture in collisions with carbon. The experimental upper limit for the cross section is $5 \mathrm{mb}$, the CDW result is $37 \mathrm{mb}$ and the IA model yields $0.23 \mathrm{mb}$. The CDW result exceeds the experimental limit almost by order of magnitude and, therefore, should be ruled out. Thus, from all the three models only the result of the IA does not contradict to the experiment. This result is, however, much smaller than the upper limit.

We have tested the sensitivity of our IA results to the form of the wavefunctions in (26) by performing calculations in which the angular correlation parameter $d$ in 26 was set to zero (i) only in the initial state, (ii) only in the final state and (iii) in both these states. (Setting $d=0$ of course also changes values of the radial correlation parameters $a$ and $b$.) The sensitivity of the cross section to the choice of the target state for a fixed state of the projectile did not exceed $20-25 \%$. At the same time when the target state was fixed but the state of the projectile was varied the change in the cross section could be as large as $\approx 100 \%^{2}$.

Using the IA model we also performed calculations in which the final state in the ion was taken as the sum of the product of two hydrogen-like $1 s$-wavefunctions with the nuclear charge equal to $Z_{p}=18$ and the correction of the first order in the electronelectron interaction. Using this perturbative approach to build the final bound state we found the cross section values which are pretty close to those obtained with the variational wavefunctions (26).

Our IA cross section $0.23 \mathrm{mb}$ is also by an order of magnitude smaller than theoretical results reported previously in $[19](\simeq 2 \mathrm{mb})$ and $[20](\simeq 3 \mathrm{mb})$. In these articles the twoelectron capture in ion-atom collisions was considered as the process of the radiative recombination of two electrons with the ion.

Two main differences between the IA model and the approach of [19] and [20] are worth to be mentioned here. Firstly, while in [19]- [20] the two electrons are assumed to

\footnotetext{
${ }^{1}$ Note that the first Born model also possesses this undesirable feature.

${ }^{2}$ The set of the IA data in figure 2 shows the largest values of the cross section.
} 
imping the ion with equal velocities, according to the IA model there exist the spread in the initial electron velocities due to the target Compton profile and this spread in the case of (the ground state of) carbon is just by a factor of 3-4 smaller than the collision velocity.

Secondly, according to the approach of [19]- [20] the neglect of the electron-electron interaction in the final state would not terminate the correlated two-electron capture provided the electron-electron interaction in the initial state is taken into account. In our IA model the electron-electron correlation in the initial state was effectively discarded when the distortion operation was performed in (21). The latter is the common assumption of the impulse approximation which assumes that the relative motion of the atomic particles in the initial state is slow enough, so that no substantial exchange of 'information' between them in this state can occur during the very short effective transition time.

Since it is stated in [19] (see also [25]) that for the radiative recombination of two equivelocity electrons with $11.4 \mathrm{Ar}^{18+}$ the electron-electron interaction in the final state is more important compared to that in the initial state, the second point can hardly account for the order of magnitude difference in the cross section ${ }^{3}$. Therefore, it is the spread in the electron velocities caused by the Compton profile of the target K-shell, which is likely to be the main reason for this difference.

\subsection{Uncorrelated two-electron capture}

The same geometric arguments show that the uncorrelated two-electron capture in collisions between a highly charged ion and a light neutral atom occurs predominantly from the atomic K-shell. Moreover, the relative role of the capture from the K-shell is now even more enhanced since one of the electrons is transferred to the ion via the Coulomb capture channel (for the Coulomb capture occurring in fast collisions the contribution from the target K-shell is known to dominate even in the case of the one-electron capture).

The cross sections for the uncorrelated two-electron capture in collisions with 11.4 $\mathrm{MeV} / \mathrm{u} \mathrm{Ar}^{18+}$ are displayed in figure 3. The uncorrelated cross section was calculated using the amplitude (25) where the initial and final one-electron states were approximated by hydrogen-like $1 s$ orbitals with the effective nucleus charge equal to $Z_{t}-0.3$ and $Z_{p}-0.3$ for the initial and final states, respectively.

Compared to the correlated capture the uncorrelated capture cross section turns out to be much larger. In addition, the uncorrelated capture cross section increases even faster with the increase of the target atomic number. Both these points take place due to the Coulomb capture channel. For the collision systems under consideration this channel is

\footnotetext{
${ }^{3}$ Taking into account the Compton profile of the target K-shell electrons, one may conclude that there is quite a low probability that in the initial channel the (vector) velocities of these electrons are equal. Both in [19] and [20] the electrons in the initial state were assumed to move with equal velocities. Since for equivelocity electrons the correlation effect should be strongest, the actual effect of the electron correlation in the initial state on the capture process was likely to be noticeably overestimated in [19] and [20].
} 


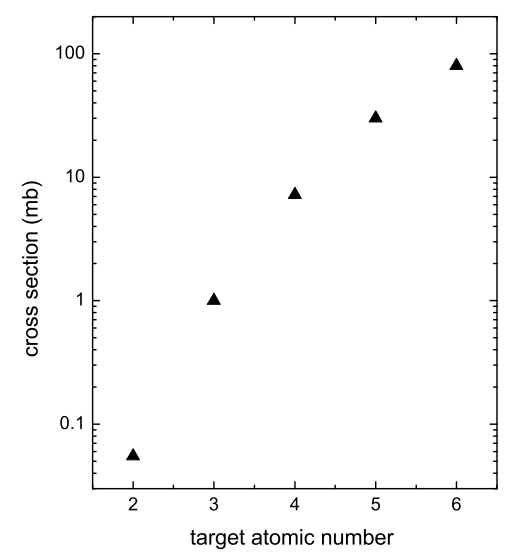

Figure 3: The cross section for the uncorrelated capture in collisions of $11.4 \mathrm{MeV} / \mathrm{u} \mathrm{Ar}^{18+}$ with different targets given as a function of the target atomic number.

quite efficient. Besides, its role strongly increases with increasing the effective charge of the target nucleus seen by the target K-shell electrons.

\subsection{Photon energy spectrum}

In figure 4 we show results for the energy spectrum of photons emitted in the correlated and uncorrelated two-electron capture processes occurring in collisions of $11.4 \mathrm{MeV} / \mathrm{u}$ $\mathrm{Ar}^{18+}$ with carbon.

According to our estimates, in collisions of $11.4 \mathrm{MeV} / \mathrm{u} \mathrm{Ar}^{18+}$ with carbon the uncorrelated capture mechanism contributes more than 99 per cent to the total two-electron capture. Besides, because of the relatively broad Compton profile of the ground state of carbon, for this collision system the two capture mechanisms produce photons which are not very well separated in the energy spectrum. The figure 4, nevertheless, suggests that a certain signature of the correlated capture can be observed in the energy spectrum of the emitted photons in the range $\omega_{k} \sim\left(v^{2}+Z_{p}^{2}\right)$ where the spectrum exhibits the bump resulting from the superposition of the contributions given by the correlated and uncorrelated capture mechanisms

One should also note that in this range of photon energies the total spectrum of photons emitted in $11.4 \mathrm{MeV} / \mathrm{u} \mathrm{Ar}^{18+}{ }_{-} \mathrm{C}$ collisions is still strongly dominated by the high-frequency tail of the one-electron radiative capture. Our calculation shows that at $\omega_{k} \sim\left(v^{2}+Z_{p}^{2}\right)$ the intensity of the photon spectrum of the one-electron radiative capture is roughly by 2 orders of magnitude larger compared to the total intensity of the photons emitted in the two-electron capture.

In the experiment [18] the photon energy spectrum and the final charge state of the projectile were measured but momenta of the target recoil ions were not detected. Under 


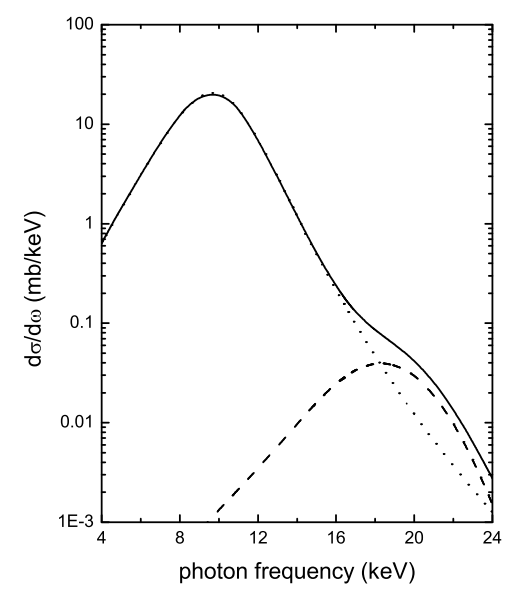

Figure 4: The spectrum of photons emitted in $\mathrm{Ar}^{18+}+\mathrm{He}\left(1 \mathrm{~s}^{2}\right) \rightarrow \mathrm{Ar}^{17+}(1 \mathrm{~s})+\mathrm{He}^{+}(1 \mathrm{~s})+h \nu$ collisions. Dash curve: the contribution of the correlated two-electron capture. Dot curve: the contribution of the uncorrelated two-electron capture. Solid curve: the sum of the correlated and uncorrelated contributions.

such conditions one can separate the one- and two-electron capture processes. However, if the photons emitted in the correlated and uncorrelated two-electron capture have a substantial energy overlap, there is no way to experimentally distinguish between the correlated and uncorrelated two-electron capture.

The uncorrelated capture mechanism was not even mentioned in [18]. However, our results show that it plays the important role in the producing of photons with energies $\omega_{k} \sim\left(v^{2}+Z_{p}^{2}\right)$. If this mechanism is taken into account than the total contribution of the above photon energy range to the two-electron capture cross section turns out to be substantially larger than just its correlated part $(0.23 \mathrm{mb})$. The magnitude of this contribution strongly depends on what particular energy is chosen as the left boundary of this photon energy range and, therefore, it is difficult to give a definite value for it. For instance, if we choose the left boundary at points $\omega_{k}=13.4$ and $14.3 \mathrm{keV}$ (where the correlated cross section is $0.25 \%$ and $0.16 \%$ of its maximum value, respectively), we get the contribution of about 2.6 and $1.4 \mathrm{mb}$. These values are already much closer to the experimental upper limit of $5 \mathrm{mb}$.

\section{Conclusions}

We have considered the two-electron capture with emission of a single photon in fast non-relativistic collisions between a highly charged ion and a light atomic target. One of the main characteristic features of this radiative process is that it is strongly dominated by the capture from the target K-shell. Two different mechanisms contribute to the two-electron-one-photon capture. 
In the uncorrelated capture one of the electrons is transferred to the ion via the interaction with the radiation field and the other electron is captured due to the Coulomb interaction with the ion. This capture mechanism produces the photon spectrum which is rather similar to that emitted in the one-electron radiative capture and has a maximum at a frequency $\omega_{k}^{(1)} \approx\left(v^{2}+Z_{p}^{2}\right) / 2$. The uncorrelated capture dominates in the total cross section for the two-electron-one-photon capture. Since the amplitude for the uncorrelated capture process is the product of the one-electron transition amplitudes, which can be evaluated with good accuracy, the theoretical description of the uncorrelated capture is rather simple.

In the correlated capture mechanism the electron-electron interaction plays the crucial role. This mechanism results in very small capture cross sections and produces the photon spectrum having a maximum at a frequency $\omega_{k}^{(2)} \approx\left(v^{2}+Z_{p}^{2}\right) \approx 2 \omega_{k}^{(1)}$.

In order to treat the correlated capture we used three different models: the first Born approximation, the Continuum-Distorted-Wave and the Impulse Approximation models. The results turned out to be extremely model-sensitive with the different models yielding cross section values which differ by orders of magnitude. From these models, only the IA was found to be consistent with the general statement that no particular structure can appear in the photon spectrum at $\omega_{k} \sim \omega_{k}^{(2)}$ if the electron-electron interaction were 'switched off'. Consequently, it is not very surprising that only the result of the IA model does not contradict to the existing experimental data for the upper limit of that part of the two-electron-one-photon capture cross section which is given by the range of $\omega_{k} \sim \omega_{k}^{(2)}$.

Our IA result is much less than the theoretical estimates of the cross section of the correlated capture obtained previously in which this process was regarded as the radiative recombination of two free electrons and an ion. The probable reason for this difference is the neglect of the target Compton profile in the previous calculations. Our IA result is also about twenty time smaller than the experimental upper limit suggesting that it might be even more difficult to observe the correlated two-electron capture process in experiment than what had been thought before.

Under collision parameters considered in this article the uncorrelated capture mechanism turned out to be by far the dominant one in the total cross section for the twoelectron-one-photon capture. Yet, the uncorrelated capture has a comparatively much stronger dependence on the target atomic number. Therefore, taking a target with the atomic number as low as possible, one can reach the collision regime where the relative role of the uncorrelated mechanism in the total two-electron capture would not be so dominant. Besides, when the target atomic number decreases the spectra of photons, emitted in the uncorrelated and correlated capture processes, narrow. This reduces their energy overlap favoring the separation of the correlated and uncorrelated capture. Note, however, that for collisions with targets having very low atomic numbers the total cross section for the two-electron capture becomes extremely small which makes it very uneasy to detect the process in experiment.

Taking into account the difficulties in the theoretical description of the correlated 
capture, very small values of the corresponding cross sections and the possible overlap between the photon spectra produced via the correlated and uncorrelated capture mechanisms, one can say that the correlated part of the two-electron radiative capture process represents the real challenge both for theory and experiment which makes this process especially attractive for explorations.

\section{References}

[1] Raisbeck G. and Yiou F., Phys.Rev.Lett., 4, 1858 (1971)

[2] Schnopper H.W., Betz H., Devaille J.P., Kalata K, Sohval A.R., Jones K.W. and Wegner H.E., Phys.Rev.Lett., 29, 898 (1972)

[3] Kienle P., Kleber M., Povh B., Diamond R.M., Stephens F.S., Grosse E., Maier M.R., and Proetel D., Phys.Rev.Lett., 31, 1099 (1973)

[4] Briggs J.S. and Dettmann K., Phys. Rev. Lett. 331123 (1974)

[5] Kleber M. and Jakubassa D.H., Nucl.Phys. A 252, 152 (1975)

[6] Shakeshaft R. and Spruch L., Phys. Rev. Lett. 38175 (1977); Rev. Mod. Phys. 51 $369(1979)$

[7] Spindler E., Betz H.-D., and Bell F., Phys.Rev.Lett., 42, 832 (1979)

[8] Anholt R. and Becker U., Phys. Rev. A 36, 4628 (1987)

[9] Eichler J., Phys.Rep. 193165 (1990)

[10] Beyer H.F. et al, J.Phys. B 26, 1557 (1993)

[11] Vane C.R., Datz S., Ditter P.F., Giese J., Jones N.L., Krause H.F., Roessel T.M., and Peterson P.S., Phys. Rev. A 49, 1847 (1994)

[12] Stölker Th. et al, Phys. Rev. A 51, 2098 (1995)

[13] Ichihara A., Shirai T., and Eichler J., Phys.Rev. A 54, 4954 (1996)

[14] Claytor N., Belkasem A., Dinneen T., Feinberg B., and Gould H., Phys. Rev. A 55, R842 (1997)

[15] Vane C.R., Krause H.F., Datz S., Grafström P., Knudsen H., Scheidenberger C., and Schuch R.H., Phys. Rev. A 62, 010701 (2000)

[16] Eichler J. and Meyerhof W.E., 1995, Relativistic Atomic Collisions (Academic Press, San Diego) 
[17] Voitkiv A.B. and Grün N., J.Phys. B 34321 (2001)

[18] Warszak A. et al, Nucl.Instr.Meth. 98303 (1995)

[19] Yakhontov V.L. and Amusia M.Ya., Phys.Rev. A 551952 (1997)

[20] Mikhailov A.I., Mikhailov I.A., Nefiodov A.V., Plunien G. and Soff G., Phys. Lett. A 328350 (2004)

[21] Dewangan D.P. and Eichler J., Phys.Rep. 24759 (1994)

[22] Belkic Dz., Journal of Com. Meth. in Sc. and Eng. 11 (2001)

[23] Abramowitz M. and Stegun I.A., Handbook of Mathematical Functions (Dover Publications, Inc., New York, 1964)

[24] Slater J.S., Quantum Theory of Atomic Structure Vol. 1 (McGraw-Hill, New York, 1960)

[25] Mikhailov A.I., a private communication. 